FACEGUK: LA CONSTRUCCIÓN SOCIAL DE LA IDENTIDAD VASCA ANALIZADA

DESDE LA ETNOGRAFÍA DIGITAL

FACEGUK: A DIGITAL ETHNOGRAPHIC APPROACH TO THE SOCIAL CONSTRUCTION OF

BASQUE IDENTITY

Koldo Díaz Bizkarguenaga

Universidad: UPV-EHU (Universidad del País Vasco - Euskal Herriko

Unibertsitatea); koldo.diaz@ehu.eus

\begin{tabular}{|c|c|}
\hline & Resumen \\
\hline $\begin{array}{l}\text { Palabras clave } \\
\text { Identidad vasca } \\
\text { Etnografía digital } \\
\text { Facebook; Territorio }\end{array}$ & $\begin{array}{l}\text { El eje principal de esta investigación es la identidad nacional de una sociedad vas- } \\
\text { ca que se ha transformado en los últimos años, en concreto, la pregunta de estudio } \\
\text { con la cual hay que abordarla y el modo en el que se construye socialmente. Para } \\
\text { ello, analizando las redes sociales, y en particular Facebook, mediante la (auto) et- } \\
\text { nografía digital, además de responder las anteriores preguntas, se ha estudiado el } \\
\text { tipo de comunidad vasca que se crea con Internet, los límites territoriales de la na- } \\
\text { ción y los agentes que influyen en estos procesos. }\end{array}$ \\
\hline
\end{tabular}

Abstract

Keywords

Basque identity

Digital ethnography

Facebook

Territory
The national identity of a recently transformed Basque society is the principal idea of this research: concretely, the research question that has to be done and the social construction processes of Basque identity. For this aim, analyzing social networks (especially Facebook) with a (auto) digital ethnographic approach, besides answering these questions, the type of Basque community that emerges with the Internet, the territorial limits of the nation and the influence of the agents that in these processes take part will be analyzed

Díaz Bizkarguenaga, Koldo (2015). FaceGUK: la construcción social de la identidad vasca analizada desde la etnografía digital. Athenea Digital, 15(2), 275-288. http://dx.doi.org/10.5565/rev/athenea.1623

\title{
Conclusiones $^{1}$
}

Al igual que hicieron Daniel Miller y Don Slater (2000) en su hoy ya clásico libro The Internet. An ethnographic approach, esta Tesis Doctoral también tengo que contarla desde el final, desde las conclusiones. El leitmotiv de la investigación fue la identidad vasca. Cuando he estado en el extranjero, debido a mi fuerte acento, me preguntaban si era español. Respondía que no. Me preguntaban si era italiano. Aunque me daban ganas de decir que sí, también respondía que no. Luego se hacía el silencio hasta que respondía que soy vasco y se volvía a crear el silencio. Entonces llegaban las preguntas y el discurso: una explicación histórica marcada con tintes políticos sobre el País Vasco, su cultura, economía y lengua. Por último, llegaba la pregunta: ¿Por qué eres vasco? Es más ¿Qué es ser vasco?

\footnotetext{
${ }_{1}$ Este artículo ha sido posible gracias a la convocatoria de ayudas para la "Contratación de Doctores recientes has ta su integración en programas de formación postdoctoral” del vicerrectorado de investigación de la UPV-EHU y se ha realizado dentro del grupo de investigación "Nor" del departamento de Comunicación Audiovisual y Publicidad
} 
Todas las respuestas conducían a los mismos lugares comunes, a los mismos callejones sin salida. Hasta que en una de estas explicaciones soporíficas intervino un amigo español. Mi amigo no conocía gran parte de su historia y, a diferencia de mí, no tenía que inventarse los datos del paro o las fechas importantes de la historia de su país. A él no le hacían preguntas, él no tenía que responder por qué es español y mucho menos qué es ser español.

\section{Del análisis de la identidad vasca al análisis de la construcción de la identidad vasca}

En el plano académico, años después, me encontré en la misma situación solo que esta conversación se dio en el congreso de la Federación Española de Sociología celebrado en Pamplona en el año 2010. Al finalizar mi comunicación sobre la juventud vasca, el casco viejo de Bilbao y la construcción de la identidad vasca los coordinadores del grupo, ambos investigadores destacados de la sociología política en España, me realizaron la misma pregunta: ¿En qué se diferencian los jóvenes vascos y los valencianos, es decir, que les hace diferentes a esos jóvenes para denominarlos vascos? He de admitir que, como investigador, cometí el error de responder desde un plano personal cuestiones académicas y que, además, cometí el error de responder una pregunta sin respuesta. Tras un debate de sordos totalmente vacío, la sesión concluyó con la afirmación de una de las coordinadoras del grupo: "en España no hay partidos políticos nacionalistas”.

Para entonces ya había comenzado con la Tesis Doctoral que iba a versar sobre la identidad vasca, los jóvenes y Facebook. La lección recibida en el congreso me sirvió para definir la investigación trasladando el objeto de estudio de la identidad vasca a la construcción de la identidad vasca. Me hice la pregunta. ¿Qué es ser vasco? No encontré respuesta y por ello decidí leer la bibliografía referente al tema. Partiendo desde Sabino Arana hasta la actualidad encontré diversas respuestas. Desde una perspectiva antropológica según la que el ser especial que a lo largo del tiempo se ha mantenido inmutable (Barandiaran, 1974), hasta una perspectiva política en la que el linaje propuesto por el fundador del Partido Nacionalista Vasco (Arana, 1980) y el hecho de vivir y trabajar en el País Vasco propuesto por la izquierda abertzale (nacionalista vasca) y analizado por Josu Amezaga (1995) eran las respuestas más repetidas. Siempre pasando por una perspectiva lingüística en la que el euskara o idioma vasco (Campión, 1985) se entendía como un elemento que define la nacionalidad vasca.

Ninguna de las respuestas fue suficiente para responder sociológicamente qué es la identidad vasca. La identidad vasca, al igual que el resto de identidades, ya no es semper idem ni idéntica: al ser ésta un constructo social, a lo largo del tiempo se ha 
transformado. Ejemplo de ello es la evolución que desde una perspectiva política ha tenido la definición de la identidad vasca. Ante la misma situación, la llegada de emigrantes españoles en busca de trabajo a las fábricas vascas, en unas pocas décadas se pasa de un discurso que excluye a ciudadanos españoles a uno que incluye a trabajadores españoles. Cuestión de clases. Lo cual recuerda la importancia de tener que analizar la identidad vasca siempre teniendo en cuenta otras identidades como la laboral y, por supuesto, la de género. Sin embargo, si vasca es aquella persona que vive y trabaja en el País Vasco ¿Qué ocurre con la diáspora? ¿Qué ocurre con aquellas personas que debido al franquismo tuvieron que dejar su país? El mismo elemento, el franquismo, elimina el euskara como posible respuesta a la pregunta "qué es ser vasco". Durante esa época mucha gente no pudo aprender ni usar el idioma y por tanto esas personas quedarían excluidas de la definición.

Una vez analizadas las posibles respuestas a la pregunta, llegué a dos conclusiones. En primer lugar, me planteé la posibilidad de que la pregunta no tuviera respuesta. Stuart Hall (1996/2003) y Judith Butler (1990/2007) me confirmaron la duda. La identidad, aunque desde una dimensión social recibe múltiples respuestas, desde un plano académico es un objeto que no es posible llenar. Es más, es precisamente por su condición performativa (Butler, 1990/2007) por la que no se puede ocupar del todo. Pero ¿Cómo realizar una Tesis Doctoral sobre un objeto que no se puede definir? El concepto de identidad bajo borradura (Hall, 1996/2003) me permitió avanzar en la investigación sin estatizar mi objeto de estudio, teniendo como base la identidad vasca pero sin olvidar que la definición propuesta no era correcta. Ésta no era más que una excusa teórica para poder llegar a mi verdadero objeto de estudio que no era otro que el análisis de la construcción de la identidad vasca.

El hecho de tener una cobertura teórica y conceptual que reforzara mi intuición sobre que la identidad no se puede definir sociológicamente me permitió llegar a una segunda conclusión. A lo mejor debía cuestionarme la propia pregunta de estudio: ¿Por qué analizar qué es ser vasco? Mi amigo español no necesitaba responder esa pregunta y mis colegas investigadores no necesitaban plantear y analizar esa pregunta. Sin embargo, yo y mis referentes teóricos vascos de otros tiempos hemos tenido la obligación científica de responder, o mejor dicho, tratar de responder qué es la identidad vasca. Àlvar Peris me explicó que:

Son los Estados-nación que podemos considerar débiles y las naciones sin estado los que más se (pre)ocupan de estas cuestiones. Por el contrario, los Estados-nación occidentales, más estables y con democracias asentadas, no se dan por aludidos y pasan de largo de estos temas (...) los ciudadanos de ese Estado-nación consolidado no se reconocen como nacionalistas, sino como 
patriotas (...) Para ello pusieron en marcha un imponente aparato institucional que consiguió que discursos marcadamente nacionalistas pasaran por no serlo (...) a la larga, (consiguen) 'naturalizar' su nacionalismo y 'objetivarlo' con la intención de que éste parezca 'normal' e inevitable a los ciudadanos (Peris, 2008, p. 1, cursivas del original).

El País Vasco carece de un estado-nación que ampare y objetive la identidad nacional ya sea en forma de un documento nacional de identidad o en forma de un examen de ciudadanía, y por tanto, hasta que esa situación se dé, la definición de la identidad vasca será una constante discusión sin fin hasta el punto de llegar a convertirse en un significante vacío (Laclau, 2005). Desde un plano social es comprensible que se siga respondiendo a esa pregunta: la sociedad y los individuos necesitamos respuestas para poder seguir adelante con nuestras vidas, necesitamos salvavidas a los que agarrarnos cuando una discusión se convierta en tempestad. Desde un plano sociológico, en cambio, es incomprensible además de incorrecto tratar de responder qué es la identidad vasca: es recorrer un camino que de antemano sabemos cómo va a terminar, es responder una pregunta mal formulada con herramientas teóricas obsoletas.

\section{Construcción de la identidad vasca desde una dimensión social y con injerencias externas}

La pregunta sociológica a responder y la vía de estudio a profundizar es ¿Cómo se construye la identidad vasca? De lo cual, al haber acaparado toda la atención académica la anterior pregunta de estudio, sabemos más bien poco. Gracias a la investigación de Alfonso Pérez-Agote (1984; 1989; 2008) seguida por Iñaki Martínez de Albéniz (2003), partiendo de la época franquista conocemos tres fases diferentes de la construcción de la identidad vasca. Esa época la denominan la sociedad sin política (Pérez-Agote, 1984; Martínez de Albéniz, 2003, p. 253): una época en la que la construcción de la identidad vasca estaba prohibida en el espacio público y, por tanto, se realizaba en un ámbito privado (como la casa o la familia). Estratégicamente, partiendo de un espacio privado la sociedad vasca conquista el espacio público dando paso a una segunda fase denominada la irrupción en la esfera pública (Pérez-Agote, 1984; Martínez de Albéniz, 2003, p. 253). Paulatinamente se da una normalización política que culmina con la aparición de los partidos políticos, los cuales, en última instancia, monopolizan la construcción de la identidad vasca hasta el punto de convertir esa época en la política sin sociedad (Pérez-Agote, 1984; Martínez de Albéniz, 2003, p. 254).

Una vez superada una pregunta de estudio que no permitía avanzar académicamente y haber explicado los motivos de ese cambio para tratar de asentar la nueva pregunta de estudio para futuras investigaciones, era el momento de empezar a anali- 
zar la construcción social de la identidad vasca a inicios del siglo XXI. El trabajo de campo me permitió conocer la importancia que la dimensión social y cultural tenían en la construcción de la identidad vasca, una identidad que en su mayor parte se ha entendido desde las ciencias sociales como política y se ha construido desde la sociedad vasca de manera negativa. La frase No somos españoles ni franceses, somos vascos que diversos movimientos sociales y políticos han utilizado en los últimos años es claro ejemplo de un modo de construir identidad por medio de una oposición a otros grupos.

Sin embargo, actualmente en la sociedad vasca los referentes identitarios relacionados con una dimensión cultural y social van cobrando fuerza. Erramun Baxok (2007) hizo que me fijara en estas dimensiones ya que, según la investigación que dirigió, la sociedad vasca cada vez identifica más lo vasco con clubes deportivos, museos o edificios y menos con ideologías o partidos políticos. Cabe pensar que la identidad vasca paulatinamente se va trasladando de la dimensión política a la social, pasando de este modo de ser una reivindicación a un sentimiento interiorizado. Al igual que la identidad española o francesa, la construcción de esta identidad nacional se convierte en cotidiana y es una práctica interiorizada por la sociedad (CEIC, 1999; 2005).

Esta investigación concreta me permitió observar que en el caso de la juventud, la construcción social de la identidad vasca se hace en gran medida desde una lógica social que tiene como eje los referentes identitarios del propio colectivo. La lógica política en la que la identidad vasca era relacionada con el nacionalismo y que tenía como referencia agentes o colectivos externos con los que trataba de diferenciarse va dejando paso a una lógica social que construye identidad vasca en las prácticas cotidianas y de manera más banal. Una joven que tomó parte en la investigación me contó que:

Es que hoy en día el contenido político (en Facebook) ha bajado bastante sobre Euskal Herria. Se habla más de la crisis de España, del gobierno de España, protestas and so on. No hay tanto contenido político.

¿Y tú publicas más sobre ese contenido político?

No. No. Hablo sobre el Athletic. (...)

$\mathrm{Y}$ yo en mi caso no me gusta hacerme fan, por ejemplo de "Bildu", fan de "Euskal presoak", fan de "EH". Pero si ves gente fan de "Bilbao", fan de "Somera". Se relaciona más con detalles cotidianos. (Nahia Galar, entrevista personal, 28 de septiembre de 2011). ${ }^{3}$

\footnotetext{
Calle del casco viejo de Bilbao con muchos bares y repleta de vida social.

3 Aunque algunas jóvenes dieron su consentimiento para incluir su nombre en la investigación, otras muchas prefirieron mantener el anonimato. En este caso, en lugar de su nombre, se utilizará un número. Cabe subrayar que
} 
El poner el acento en las prácticas de la identidad en vez de en la definición de la identidad me permitió entender a los sujetos que estaban detrás de estas prácticas como ciudadanos y no como nacionalistas. De este modo pude comprender que la ciudadanía vasca construye la identidad vasca de múltiples formas: por ejemplo, mediante el euskara $y$ mediante su lugar de residencia. Por tanto, las anteriores respuestas que no valían para definir qué es la identidad vasca, son la respuesta idónea para entender cómo se construye la identidad vasca. La diferencia radica en la pregunta y en la respuesta que ésta exige. Al preguntar por la definición, la respuesta ha de ser éste $o$ aquel elemento: las respuestas son excluyentes entre sí y siempre van a dar una respuesta parcial que excluya a parte de la ciudadanía vasca. Al preguntar por los modos de construcción, la respuesta ha de ser éste $y$ aquel elemento: las respuestas permiten incluir otras respuestas y van a dar una respuesta parcial que incluya al resto de la ciudadanía. El cambio de pregunta de estudio y el ver la identidad vasca más allá de un prisma político que no solo tuviera en cuenta la condición de que el País Vasco es una nación sin estado me permitió observar la importancia que la dimensión social y cultural y las relaciones endogrupales tienen en la construcción de la identidad vasca.

Sin embargo, no hay que olvidar que esta condición influye en la construcción de la identidad vasca. La injerencia externa en el análisis de la identidad vasca la podemos encontrar en la misma pregunta de estudio que la academia ha seguido para analizar este objeto. La pregunta de estudio, además de ser una motivación propia, ha sido propuesta y una y otra vez exigida desde la academia española. Mientras se cuestiona muy poco la identidad española se piden respuestas sobre la identidad vasca. Una vez más, (la falta de) un estado que (no) ampare a la ciudadanía y a la identidad nacional marca la diferencia.

En cuanto a la construcción de la identidad vasca, uniendo el plano político y el social, las tres diferentes administraciones influyen en la capacidad de construcción de la identidad vasca. El caso más claro es el del euskara. La Comunidad Autónoma Vas ca, la Comunidad Foral de Navarra y el estado francés llevan tres políticas lingüísticas diferentes en el País Vasco, lo cual, influye en el uso y en la importancia que la ciudadanía vasca otorga a este elemento dependiendo del lugar de residencia. Por lo tanto, en el caso de la identidad vasca, ésta identidad colectiva se construye por las relaciones endogrupales y por la oposición a otros grupos, pero también la construye un agente externo. Mediante leyes otros grupos limitan unos referentes identitarios que influyen en los modos de construir la identidad vasca. De este modo, la construcción pasa de ser cosa de los nacionalistas vascos a ser una cuestión de toda la ciudadanía vasca. Pero también está en manos de los nacionalistas españoles y franceses.

las entrevistas se realizaron en euskara y que en este artículo se ha optado por traducir las entrevistas personales al castellano. 


\section{Facebook, un espacio privilegiado aunque no seguro para la construcción social de la identidad vasca}

La influencia del estado español y francés en la construcción de la identidad vasca también se da en una dimensión social y en las relaciones sociales mediadas por Internet. Los jóvenes que tomaron parte en la investigación señalaron que no podían registrarse en Facebook como vascos, tenían que hacerlo como ciudadanos españoles o franceses. Tal y como Àlvar Peris explica: "los estados nación todavía ejercen una capacidad de control mayor sobre las nuevas tecnologías de lo que a muchos les gustaría” (2010, p. 229).

Las limitaciones administrativas para la construcción social de la identidad vasca se trasladan al espacio online. Pero además, debido a la lógica propia de Internet, estas limitaciones adquieren nuevas formas. Muchos jóvenes expresaron su temor por compartir ciertas opiniones y por construir la identidad vasca a través de Facebook de una manera determinada ya que se sentían observados. La idea de panóptico digital (boyd, 2002) ayuda a entender como esa sensación de supervisión constante hace que los jóvenes no habiten un espacio como a ellos les gustaría y que, por tanto, se autocensuren ciertas prácticas.

Facebook no es un espacio seguro para la construcción de la identidad vasca, pero sin embargo si es un espacio privilegiado. William Dutton (2009) explica el papel que juega Internet para la sociedad por permitir prácticas que pueden superar la lógica administrativa política clásica. En cualquier momento y superando las distancias geográficas pueden acceder a ese espacio y encontrarse con sus amigos. Además, pueden construir la identidad vasca mediante texto escrito, fotos y vídeos y mediante la adscripción a grupos: una multiplicidad de opciones que supera la fase de la política sin la sociedad en la que las instituciones políticas tenían el monopolio de la construcción de la identidad vasca. A través de Internet la sociedad vasca puede construir la identidad vasca más allá de las lógicas de las instituciones. Si bien desde un plano sistémico los usuarios tienen que registrarse como españoles o franceses, aprovechándose del propio sistema, los jóvenes crean vías para no verse obligados a ello. La observación participante me ayudó a identificar y a entender los numerosos jóvenes que tenían como lugar de nacimiento islas y países lejanos al País Vascos. La pista final me la dio un joven cuyo lugar de nacimiento era "Durango, México": su pueblo natal era Durango, un pueblo de Bizkaia, pero como al elegir esta opción debía marcar "Durango, España" prefirió ser ciudadano de México.

Al igual que en la época franquista, la sociedad también construye la identidad vasca desde un espacio privado: en este caso, desde una empresa privada como Face- 
book. Pero la lógica o la intención de la sociedad no es conquistar el espacio público, ya que éste ya fue conquistado y ya que fueron desplazados de ése espacio. La estrategia de ocupar el espacio público desde el espacio privado deja paso a la táctica (De Certau, 1996) de hacer propio y habitable el espacio privado: de manera reflexiva los jóvenes se apoderan de los límites y las limitaciones de Facebook y es desde ahí y para ahí donde construye la identidad vasca.

\section{La sociedad vasca del "dosel sagrado" a la "nación multipolar"}

Tengo que volver al inicio. A la pregunta de estudio. Las respuestas sobre la definición de la identidad vasca daban una visión de la sociedad vasca excluyente y de una única respuesta. Esa respuesta a la definición, ese elemento único de la identidad vasca se entendió como un dosel sagrado (Gurruchaga, 1990) sobre el cual se articulaba y organizaba la sociedad vasca: una sociedad, que al igual que las tribus primitivas, gira en torno a un tótem.

Al cambiar la pregunta de estudio encontramos múltiples formas de construir la identidad vasca y, por tanto, múltiples referentes identitarios sobre los que la sociedad vasca se cimenta. La idea de "nación multipolar" (Anduaga, 2011) ayuda a entender los múltiples grupos a los que los jóvenes se adscriben en Facebook: el "rito de paso" para acceder a un colectivo es un "clic" de botón, lo cual permite que la flexibilidad para entrar y salir del grupo sea mayor y que las adscripciones a otros grupos sean posibles. A diferencia de los grupos cara a cara, los jóvenes pueden tomar parte en más de un grupo relacionado con la identidad vasca y no han de elegir entre uno u otro elemento: pueden participar en un grupo de un partido político y también en un grupo relacionado con el euskara y también en un grupo relacionado con la cultura vasca.

Dentro de la misma comunidad aparecen múltiples colectivos que tienen como eje diferentes referentes identitarios. El dosel sagrado se cae, deja de ser un dosel y se multiplican los elementos sobre los cuales versar sin que ello suponga ningún tipo de contradicción. Además, el dosel sagrado lo derriba la sociedad vasca al reírse de los referentes identitarios sobre los que construye la identidad vasca. Un claro ejemplo de ello es el grupo de Facebook "Euskalduna naiz profileko argazkian mendian ez agertu arren" ("Soy vasco aunque en la foto de perfil no aparezca en el monte"). Durante el franquismo el monte, al igual que la casa, fue un espacio privilegiado y seguro para construir la identidad vasca lejos de los oídos del régimen. La naturaleza juega un papel vital en el imaginario colectivo de una nación (Guibernau, 2009) e ir al monte fue, y sigue siendo, una vía de escape y una práctica de ocio arraigada en el País Vasco. Hasta el punto que hoy en día muchos jóvenes ponen fotos suyas en el monte como foto de perfil de Facebook y otros se ríen de ello. 
Cambiando la pregunta de estudio puede observarse una sociedad con múltiples referentes identitarios, donde los diferentes colectivos que en torno a estos elementos se crean tienen cabida dentro de una misma comunidad. Aplicando la nueva pregunta de estudio al espacio de Internet, pude encontrar una sociedad que construye su identidad nacional fuera de los límites geográficos.

\section{El país digital: el territorio en la era de Internet}

La identidad nacional se ha construido hasta ahora en un lugar geográfico: en un estado-nación, en una nación o en diferentes países debido a la diáspora. Internet no ocupa un espacio geográfico y por ello al hablar de la construcción de la identidad vasca en Facebook siempre he subrayado que ésta se realiza en un espacio. Un espacio de flujos (Castells, 2012), si se quiere.

Los estudios sobre Internet y transnacionalismo (Vertovec, 2001) han explicado cómo ciudadanos fuera de sus países construyen su identidad nacional a través de Internet, entendiendo Internet como una herramienta. Pero Internet, además de ser una herramienta es una espacio: un espacio en el que, aunque no sea cara a cara, la ciudadanía se encuentra y habita. Este es el reto teórico que quiero proponer mediante la idea de país digital: ¿Qué supone y qué consecuencias tiene el hecho de que la identidad nacional no se construya en un lugar geográfico?

El lugar geográfico y el espacio online convergen a modo de Hiperespacio (Gómez, 2002) creando un territorio donde la ciudadanía se encuentra cara a cara y en Internet, en su país y fuera de su país. Un concepto, el de territorio, que tiene en cuenta los límites geográficos de un país y sus limitaciones administrativas y, también, los límites de una comunidad o nación que las relaciones sociales y los símbolos dibujan. Por un lado, la identidad vasca se construye en un lugar geográfico, en una nación sin estado propio donde dos estados limitan ésta construcción. El mapa se supera pero no se borra, ya que aunque Internet no ocupe un lugar geográfico sí se ve influenciado por el mismo. Por otro lado, la identidad vasca se construye a través de Facebook, a través de Internet: un espacio que aunque converja con el espacio offline, dispone de su propia arquitectura y su propia lógica.

Con Internet surge un territorio donde la ciudadanía construye identidad nacional fuera de sus fronteras y de cualquier frontera geográfica. En la actualidad la sociedad vasca separada en dos estados se encuentra en Internet: un espacio que habita con diferentes influencias administrativas y un espacio donde construye identidad nacional de diferente manera dependiendo, entre otras cosas, del lugar de residencia. En Inter- 
net, la sociedad vasca superando los límites geográficos y las fronteras impuestas puede compartir sus referentes identitarios y habitar su propio territorio.

Esto fue lo que me enseñaron los jóvenes del grupo Erasmus con los que realicé la etnografía digital.

\section{Metodología}

El objetivo es analizar la construcción social de la identidad vasca a través de y en Facebook. ¿Cómo lo hago? ¿Por dónde empiezo? Pues por un análisis cuantitativo: analizando los datos que diferentes organismos (básicamente, Eusko Ikaskuntza, Gobierno Vasco y el Observatorio Vasco de la Juventud) publicaban sobre el uso de Internet y la identidad vasca. Un primer paso. Contando cuántos vascos hay en Facebook no podía responder cómo construyen la identidad vasca, pero ante un objeto de estudio y un espacio poco estudiados de algún modo debía empezar a andar.

Pasé a las técnicas cualitativas. Quería recabar discursos de jóvenes que me explicaran qué referentes y prácticas mantenían para la construcción social de la identidad vasca en Facebook. No resultó. Realicé diversas entrevistas y grupos de discusión a jóvenes que cumplían diferentes variables (edad, género, lugar de residencia (no) vasco [relacionado al entorno institucional y político] y grado de uso de las TIC y en especial de Facebook) pero la mayoría de las conversaciones terminaban igual que las que mantuve en el extranjero o en el congreso de la FES: los jóvenes acababan por decirme qué era para ellos la identidad vasca. Algo muy interesante pero que no se ajustaba a mi objetivo principal. Accedí al juego. Les pregunté directamente que era para ellos la identidad vasca. En un primer momento me respondían uno de los elementos anteriormente citados, les respondía, lo cuestionaba y se hacía el silencio. Ahora era yo el extranjero, ahora era yo quien hacía las preguntas sin respuesta. ¿Por qué?

La respuesta de una de las jóvenes me abrió los ojos:

Paso porque no sé responder. O sea, es una cosa que lo he hablado bastantes veces, con mucha gente, que al principio tenía en la cabeza una cosa, pues, lo que podía ser la identidad vasca o que nos han metido que puede ser o que me han dicho. Pero luego ha ido cambiado y en vez de ir definiéndose he ido teniendo cada vez más dudas. ¿Entiendes? Así que... ¡Buf! (Entrevista № 1, 3 de agosto de 2010).

La mayoría de los jóvenes, al igual que yo, querían pero no podían responder qué es la identidad vasca. Pero esta joven ni lo quería, ni lo necesitaba. Aunque en un plano teórico yo también tenía claro que no se podía responder qué es la identidad vasca, 
les pregunté a los jóvenes por ello. En el plano teórico había superado la necesidad de buscar una definición, pero en el plano empírico y práctico no: la investigación estaba descompensada y no adquiría respuestas para la nueva pregunta de estudio.

Entonces conocí la etnografía virtual (Hine, 2004). Empecé por observar a mis amigos de Facebook explicándoles que estaba haciendo una investigación. Busqué modos de construir identidad vasca en Facebook por medio de la observación participante y luego contacté con algunos jóvenes que mantenían estas prácticas para realizar entrevistas en profundidad: me encontré con jóvenes "militantes" que utilizaban Facebook "no para jugar a Farmville, para ideologizar a sus amigos" (Oier Azkarraga, 27 de septiembre de 2011), con jóvenes que, al igual que yo, unían Facebook y la identidad vasca de manera "utilitarista" para sus fines personales (en su gran mayoría músicos, escritores y periodistas), me encontré con no tan jóvenes que de una manera "banal" construían la identidad vasca y donde los grupos como el anteriormente mencionado Euskalduna naiz profileko argazkian mendian ez agertu arren cobraron una vital importancia y, por último, me encontré con jóvenes que construían la identidad vasca desde "el extranjero".

En el año 2010 tuve la oportunidad de realizar una estancia de investigación en el grupo "Mediacciones" de la UOC con la profesora Elisenda Ardèvol. Gracias a ella y a Edgar Gómez pude diseñar una "etnografía digital" (ya que lo virtual se convertía en real e iba a superar el espacio online) en la que el elemento básico era introducirme en un grupo. Suerte, paciencia y tiempo... ¿Ciencia? Una de las jóvenes que entrevisté porque se encontraba continuamente viajando y me ayudaba a entender la influencia que el lugar de residencia tiene en la construcción de la identidad vasca por la importancia que se le otorga a diferentes símbolos dependiendo del lugar, me contó que viajaba tanto por una beca que tenía. Junto con otros 40 jóvenes se encontraba a lo largo y ancho del mundo conociendo diferentes realidades empresariales y como estos jóvenes hicieron muy buena relación durante sus clases presenciales en el País Vasco decidieron hacer un grupo privado de Facebook para hablar de temas de la beca y para mantener sus raíces vascas.

Pedí permiso a todos los miembros del grupo para acceder. Entré. Observé: analicé qué referentes identitarios manejaban, de qué hablaban, cómo y en qué idioma. Al de unos días participé: seguí las conversaciones, inicié nuevas, hice preguntas, respondí las que sabía. Por último, identifiqué a los miembros más activos del grupo y realicé con siete jóvenes entrevistas en profundidad. La distancia geográfica hizo que Skype fuera el mejor modo de realizar dichas entrevistas. Les di a elegir si querían hacer la entrevista vía video llamada, video llamada sin imagen o vía chat y si la querían hacer en euskara o castellano. Todos los jóvenes eligieron la opción del chat y todos los que 
sabían euskara (seis de siete) optaron por hacer la entrevista en euskara. Al de nueve meses y con mucha información que interpretar me despedí y abandoné el grupo compartiendo con ellos lo que había escrito hasta el momento (cuando deposité la Tesis Doctoral les envíe una copia de todo lo que me habían ayudado a comprender)

En este proceso de adaptación al grupo me ayudó mucho el hecho de que yo también estaba en el extranjero realizando estancias de investigación en diferentes universidades. Me di cuenta de la situación y aproveché la ocasión llevando a cabo un diario que me ayudara a entender mi uso de Facebook y mis relaciones a través de Facebook. Es así como también realicé una autoetnografía durante mis estancias investigadoras en Ottawa, Oxford y Buenos Aires.

\section{Introducción y futuras líneas de investigación}

La Tesis Doctoral, tal y como la evolución metodológica refleja, ha sido un camino en el que la oscuridad del objeto de estudio y del espacio no me permitía ver el siguiente paso a dar y mucho menos el final del camino. El proceso de investigación ha sido como un bosque donde cualquier ruido ajeno, en forma de aportación o crítica al trabajo, te asusta y donde el objeto que ves está borroso y con muchas sombras. Han aparecido atajos en el bosque, en forma de salidas rápidas a cuestiones difíciles. Han aparecido cazadores que lo único que querían era que acabara esta investigación y cogiendo sus atajos.

El hecho de tener como brújula un objetivo general que me orientase en cada decisión fue determinante. Cuando estaba perdido miraba el papel y veía que mi objetivo era responder cómo se construye la identidad vasca a través de y en Facebook. No definir la identidad vasca, no convertirme en doctor y algún día en profesor. Gracias a mi objetivo, y por supuesto, a toda la gente que me acompañó en el viaje, pude iniciar un nuevo camino metodológico en la academia vasca (pude realizar la primera etnografía digital en el País Vasco) y pude con mis conclusiones aportar algo de conocimiento al estudio de la identidad vasca, su construcción social y Facebook.

Hay vida después de la Tesis Doctoral, sí. Una vida llena de preguntas. En mi caso, la pregunta a responder es sobre el territorio que el País Vasco habita en la era de Internet. Sobre el territorio donde la ciudadanía vasca se encuentra por encima de los límites geográficos de los estados español y francés y sobre el país digital que la construcción online de la identidad vasca dibuja. 


\section{Referencias}

Amezaga, Josu. (1995). Herri kultura: euskal kultura eta kultura popularrak. Leioa: Servicio editorial UPV-EHU.

Anduaga, Uxoa. (2011). La risa (no) redentora. Ensayo sobre el humor y la construcción de la realidad social. Telondefondo, 11, 1-15.

Arana, Sabin. (1980). Obras completas de Arana-Goiri'tar Sabin (Sabino de Arana-Goiri). Donostia: Sendoa.

Barandiaran, Joxe Migel. (1974). Euskalerriko leen-gizona, Obras completas (XI). Bilbao: La gran enciclopedia Vasca.

Baxok, Erramun; Etxegoin, Pantxoa; Lekunberri, Terexa; Martínez de Luna, Iñaki; Mendizabal, Larraitz; Ahedo, Igor; Itzaina, Xabier, \& Jimeno, Roldán (2007). Euskal nortasuna eta kultura XXI. mende hasieran. Donostia: Eusko Ikaskuntza.

Boyd, Danah. (2002). Faceted Id/entity: Managing Representation in a Digital World. Tesis Doctoral sin publicar, MIT.

Butler, Judith. (1990/2007). El género en disputa: el feminismo y la subversión de la identidad. Barcelona: Paidós.

Campión, Arturo. (1985). El idioma y la libertad de los pueblos, Obras completas (XV). Iruñea: Mintzoa.

Castells, Manuel. (2012). Networks of Outrage and Hope: Social Movements in the Internet Age. Madrid: Alianza.

CEIC, (1999). Institucionalización política y reencantamiento de la sociedad: las transfromaciones en el mundo nacionalista. Donostia: Servicio central de publicaciones del gobierno vasco.

CEIC (2005). Hacia una nueva cultura de la identidad y la política: tendencias en la juventud vasca. Donostia: Servicio central de publicaciones del gobierno vasco.

De Certeau, Michel. (1996) La invención de lo cotidiano I. Artes de hacer. México: Universidad Iberoamericana.

Dutton, William. (2009). The fifth Estate emerging through the network of networks. Prometheus, 27(1), 1-15. Doi:10.1080/08109020802657453.

Gómez, Edgar. (2002). Espacio, Ciberespacio e Hiperespacio: Nuevas configuraciones para leer la Comunicación Mediada por Computadora. Archivo del Observatorio para la CiberSociedad. Retrieved from: http://www.cibersociedad.net/archivo/articulo.php?art=19

Guibernau, Montserrat. (2009). La identidad de las naciones. Barcelona: Ariel.

Gurruchaga, Ander. (1990). La refundación del nacionalismo vasco. Leioa: Servicio editorial UPV-EHU.

Hall, Stuart. (1996/2003). ¿Quién necesita identidad? En Paul Du Gay y Stuart Hall (Eds.), Cuestiones de identidad cultural (pp. 13-39). Buenos Aires: Amorrortu.

Hine, Christine. (2004). Etnografía Virtual. Barcelona: Servicio editorial UOC.

Laclau, Ernesto. (2005). La razón populista. México: Fondo de culturaeconómica. 
Martínez de Albéniz, Iñaki. (2003). La poética de la política: usos de la política en el País vasco. Leioa: Servicio editorial UPV-EHU.

Miller, Daniel \& Slater, Don. (2000). The Internet. An ethnographic approach. Oxford: Berg.

Pérez-Agote, Alfonso. (1984) La reproducción del nacionalismo. El caso vasco. Madrid: CIS.

Pérez-Agote, Alfonso. (1989) Sociología del nacionalismo. Erandio: Eusko Jaurlaritza.

Pérez-Agote, Alfonso. (2008) Las raíces sociales del nacionalismo vasco. Madrid: CIS.

Peris, Àlvar. (2008). Identidad nacional, televisión y vida diaria: perspectivas para un debate del caso español. En María Encarna Nicolás Marín \& Carmen González Martínez (Eds.), Ayeres en discusión. Temas clave de historia contemporánea hoy (pp. 1-20). Murcia: servicio publicaciones Universidad de Murcia.

Peris, Àlvar. (2010). Internet e identidad nacional: Estado, dominios y comunidades virtuales. IC-Revista Científica de información y comunicación, 7, 221-253.

Vertovec, Steven. (2001). Transnationalism and identity. Fournal of Ethnic and Migration Studies, 27(4), 573-82. http://dx.doi.org/10.1080/13691830120090386

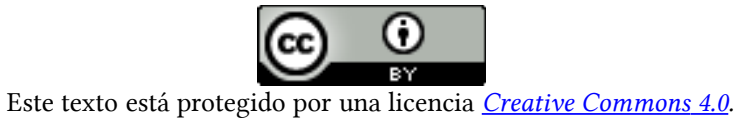

Usted es libre para Compartir — copiar y redistribuir el material en cualquier medio o formato- y Adaptar el documento - remezclar, transformar y crear a partir del material - para cualquier propósito, incluso comercialmente, siempre que cumpla la condición de:

Atribución: Usted debe reconocer el crédito de una obra de manera adecuada, proporcionar un enlace a la licencia, e indicar si se han realizado cambios . Puede hacerlo en cualquier forma razonable, pero no de forma tal que sugiera que tiene el apoyo del licenciante o lo recibe por el uso que hace. 